Published in final edited form as:

Infant Behav Dev. 2008 September ; 31(3): 481-487. doi:10.1016/j.infbeh.2007.12.004.

\title{
Breastfeeding and Antidepressants
}

\author{
Tiffany Field ${ }^{1,2}$ \\ 1 Touch Research Institutes, University of Miami School of Medicine
}

2 Fielding Graduate University

\begin{abstract}
Although a large literature supports the benefits of breastfeeding, this review suggests that breastfeeding is less common among postpartum depressed women, even though their infants benefit from the breastfeeding. Depressed mothers, in part, do not breastfeed because of their concern about potentially negative effects of antidepressants on their infants. Although sertraline (Zoloft) and paroxetine (Paxol) concentrations are not detectable in infants' sera, fluoxetine (Prozac) and citalopram (Celexa) do have detectable levels. Unfortunately these findings are not definitive because they are based on very small sample, uncontrolled studies. As in the literature on prenatal antidepressant effects, the question still remains whether the antidepressants or the untreated depression itself has more negative effects on the infant. It is possible that the positive effects of breastfeeding may outweigh the positive effects of the antidepressants for both the mother and the infant. In addition, some alternative therapies may substitute or attenuate the effects of antidepressants, such as vagal stimulation or massage therapy, both therapies being noted to reduce depression. Further studies of this kind are needed to determine the optimal course of therapy for the benefit of the depressed, breastfeeding mother and the breastfed infant.
\end{abstract}

Breastfeeding has been noted to have significant benefits for both mothers and infants. Despite these benefits, postpartum depressed mothers are less likely to breastfeed, and when they do, the breastfeeding is for shorter periods of time (Field, Hernandez-Reif \& Feijo, 2002; Galler, Harrison, Biggs, Ramsey \& Forde, 1999). One of the factors that likely affects depressed mothers' decision to not breastfeed is the concern about the potential negative effects of antidepressants on their breast milk and, in turn, on their infants' development. In this paper, recent literature on breastfeeding and antidepressants is reviewed, and suggestions are made for future research.

\section{Breastfeeding Benefits}

Although breastfed newborns have been noted to be more irritable, they have also shown more physiological (DiPietro, Larson \& Porges, 1997; Zeskind, Marshall \& Goff, 1992) and neurobehavioral organization (Hart, Boylan, Carroll, Musick \& Lampe, 2003). Later in infancy, they are reputedly more active and show easier temperament including less irritability and greater sociability (Field et al., 2002; VanDiver, 1997; Worobey, 1998). This may, in part, be related to the mothers of breastfed infants showing more visual gaze and tactile stimulation of their infants (Kuzela, Stifter \& Worobey, 1990; Lavelli \& Polli, 1998). The better motherinfant interactions and the easier infant temperament may contribute to the breastfed infants' superior cognitive and motor development (Sacker, Quigley \& Kelly 2006) that, in turn, results

\footnotetext{
Publisher's Disclaimer: This is a PDF file of an unedited manuscript that has been accepted for publication. As a service to our customers we are providing this early version of the manuscript. The manuscript will undergo copyediting, typesetting, and review of the resulting proof before it is published in its final citable form. Please note that during the production process errors may be discovered which could affect the content, and all legal disclaimers that apply to the journal pertain.
} 
in better performance on cognitive tasks in infants (Gerrish \& Mennella, 2000) and higher IQ scores in childhood (Hoffman, Birch, Birch, Uauy, Castaneda, Lapus \& Wheaton, 2000).

In addition, prolonged breastfeeding has been associated with less bedwetting in childhood (Barone, Ramasamy, Farkas, Lerner, Creenan, Salmon, Tranchell, Schneider; 2006), less obesity in childhood (Grummer-Strawn \& Mei, 2004; Shields, O'Callaghan, Williams, Najman $\&$ Bor, 2006), enhanced immune function (Jackson, Nazar, 2006) and, not surprisingly then, less health problems and fewer doctor visits (Oddy, Scott, Graham, Binns, 2006). Some have attributed the benefits of breastfeeding to components in the breast milk, for example, the higher levels of docosahexaenoic acid (DHA) in breast milk (Hart, Boylan, Carroll, Musick, Lampe, 2003).There are, of course, many other possible underlying mechanisms, e.g. the greater amount of pressure receptor stimulation of the intraoral cavity of breastfed infants, stimulation noted to increase vagal activity (Field, Hernandez-Reif \& Feijo, 2002) and the release of oxytocin (Uvnas-Moberg \& Petersson, 2005), both of which may lead to better development.

\section{Incidence of Breastfeeding in Depressed Women}

Although the overall incidence of breastfeeding has increased $15 \%$ over the last two decades (Labbok, Wardlaw, Blanc, Clark, Terreri, 2006), the incidence of breastfeeding among postpartum depressed women is significantly lower in many countries. For example, in Pakistan, 38\% of women who had high depression scores stopped breastfeeding whereas $62 \%$ of women with low depression scores continued breastfeeding (Taj \& Sikander, 2003). Of the non-breastfeeding mothers, $37 \%$ reported that their depressive symptoms preceded their discontinuing breastfeeding. In a study in Australia, of the $18 \%$ of women diagnosed with postpartum depression, the onset occurred before 2 months in $63 \%$ of cases (Henderson, Evan, Straton, Priest \& Hagan, 2003). The median duration of breastfeeding was 26 weeks for women with early-onset depression, 28 weeks for women with late-onset depression, and 39 weeks for women without depression. After controlling for potential confounds, the early cessation of breastfeeding was significantly associated with postpartum depression. Similar data have been reported in the US. For example, in a study in Oregon, there was an inverse relationship between depressive symptoms and breastfeeding at six weeks postpartum, but curiously not at twelve weeks (Hatton, Harrison-Hohner, Coste, Dorato, Curet \& McCarron, 2005).

\section{Breastfeeding Benefits for Depressed Mothers and their Infants}

Although postpartum depressed mothers choose not to breastfeed or do so for relatively short periods of time (Field et al., 2002; Galler et al., 1999), at least one study has been conducted on breastfeeding by depressed mothers, and, in that study, the infants significantly benefited (Jones, McFall \& Diego, 2004). The one-month-old infants of depressed mothers who had stable breastfeeding patterns were less likely to have reactive temperaments, and at one month, they did not show the abnormal frontal EEG asymmetry patterns that are typically seen in infants of depressed mothers. In addition, they went on to have better mother-infant interactions when the infants were 3-months-old. Thus, the depressed mothers and infants benefited from breastfeeding, although in this study it was not clear whether the mothers were taking antidepressants. Curiously, antidepressants were never mentioned in the Jones et al. (2004) study. The benefits they reported may not pertain to infants of depressed mothers who are taking antidepressants.

\section{Measuring Drug Concentrations}

In most of the studies on antidepressants, maternal and infant serum and breast milk have been analyzed using liquid chromatography/mass spectrometry methods (Berle, Steen, Aamo, Breilid, Zahlsen \& Spigset, 2004). A recent paper detailed a novel method for quantifying the concentrations of multiple selective serotonin reuptake inhibitors (SSRIs) and tricyclic 
antidepressants in breast milk. The method consisted of common liquid/liquid and solid phase extraction followed by HPLC separation on a common column and UV detection (Hostetter, Stowe, Cox \& Ritchie, 2004). The authors showed the method to be highly accurate and a precise technique for measuring multiple antidepressants simultaneously.

\section{Breastfeeding and Antidepressants}

Several reviews have been conducted on breastfeeding and antidepressants. One review focused on the use of fluoxetine, sertraline, paroxetine, fluvoxamine and citalopram, which were commonly used antidepressants at that point in time, and all belong to the selective serotonin reuptake inhibitor group of antidepressants (Misri \& Kostaras, 2002). At the time of their review, there were no published controlled studies on the use of antidepressants by breastfeeding women, so the review was based on published individual case reports, case series and pharmacokinetic investigations. The authors noted that no serious adverse events had been reported on infants exposed to antidepressant medications through breast milk. In a more recent review, the authors concluded that relatively few serious adverse effects have been reported (Whitby \& Smith, 2005). They noted that, although tricyclic antidepressants had been the treatment of choice in the past, the selective serotonin reuptake inhibitors are gaining popularity, reputedly due to their "superior safety profiles". They also noted that sertraline was the most prescribed antidepressant and had not had any reported adverse effects. However, the authors suggested that the most practical approach may be to have depressed mothers continue therapy with the previously effective drug.

In another recent review of eleven studies (examining a total of 306 children), no impairment of infant neurodevelopment was noted following exposure to SSRIs, although the author suggested that two studies (examining 81 children) had unwanted effects of SSRI exposure, but the exposure had occurred during the fetal period (Gentile, 2005a). The unwanted effects included subtle motor development and motor control problems. In a similar, but more cautious review the same author advised that at present compounds such as bupropion, mirtazapine and reboxetine "should not be used as first-line agents in the pharmacological treatment of depression in pregnancy and breastfeeding" (Gentile, 2005b)

\section{Sertraline (Zoloft): The Most Prescribed Antidepressant}

As already mentioned, sertraline is noted to be the most prescribed antidepressant with no adverse effects (Whitby \& Smith, 2005). In one study comparing sertraline with the other SSRIs, sertraline and paroxetine were not detected in any of the drug-exposed infants' serum (Berle et al., 2004).

Using a different methodology, platelet levels of serotonin (5-HT) were measured in breastfeeding mother-infant pairs before and after six-sixteen weeks of maternal treatment with sertraline for major postpartum depression (Epperson, Czarkowski, Ward-O'Brien, Weiss, Gueorguieva, Jatlow \& Anderson, 2001). Mothers receiving clinical doses of sertraline experienced blockade of the platelet 5-HT (serotonin) transporter. However, platelet 5-HT uptake in the breastfeeding infants of the treated mothers was unaltered. This study suggested that while the SSRI sertraline decreases the serotonin levels in the mother, breastfeeding does not affect the peripheral or central serotonin transport in their infants. However, this study suffered from a small sample size $(\mathrm{N}=11)$. In a slightly larger sample $(\mathrm{N}=15$ women and 182 breast milk samples), the highest concentrations were observed in the hindmilk 8 to 9 hours after maternal ingestion of sertraline (Stowe, Hostetter, Owen, Ritchie, Sternberg, Cohen \& Nemeroff, 2003). These results suggested that discarding the breast milk nine hours after the maternal dose then decreased the infant daily dose of sertraline by a mean of $17 \%$ and, again, no adverse events were reported or documented in any of the infants. 
A good example of the methodological problem of limited sample size is illustrated by a study that conducted high performance liquid chromatography on maternal and infant serum concentrations of sertraline, paroxetine and fluvoxamine (Hendrick, Fukuchi, Altshuler, Widawski, Wertheimer \& Brunhuber, 2001). The authors reported that there was no detectable medication present in any infant exposed to paroxetine $(\mathrm{N}=16)$ or fluvoxamine $(\mathrm{N}=4)$.

However, among the infants exposed to sertraline $(\mathrm{N}=30)$, detectable medication was present in $24 \%$ of the serum samples. The fact that the sertraline-infants came from a sample twice to seven times the size of the other samples could explain this finding. Despite the greater detectability of sertraline, none of the infants in the study experienced adverse sequelae.

\section{Paroxetine (Paxol)}

Paroxetine is said to be found in higher concentrations in infant milk and serum than sertraline, but in lower concentrations than fluoxetine and citalopram (Ohman, Hagg, Carleborg \& Spigset, 1999). In this study, the paroxetine concentration in hindmilk was $78 \%$ higher than in foremilk, not unlike the hindmilk/foremilk difference found for sertraline (Stowe et al., 2003). However, the mean estimated dose the infants experienced ranged from .7\% to $2.9 \%$ of the weight-adjusted maternal dose, suggesting that they were being exposed to very low levels. Once again, no adverse drug reactions or unusual behaviors were reported for the infants. However, the sample was again, very small $(\mathrm{N}=6)$.

In a much larger sample $(\mathrm{N}=108)$, breast milk and paired maternal and infant sera were analyzed by high-performance liquid chromatography with ultraviolet detection and a limit detection of 2 ng/ml (Stowe, Cohen, Hostetter, Ritchie, Owens \& Nemeroff, 2000). The paroxetine concentrations were highly variable $(2-101 \mathrm{ng} / \mathrm{ml})$ and were present in all breast milk samples. Once again, greater concentrations were found in the hindmilk than in the foremilk. The authors concluded that these data parallel the available data on other selective serotonin reuptake inhibitors.

In a similar study, the concentrations of paroxetine were determined using gas chromatography/ mass spectrometry (Misri, Kim, Riggs \& Kostaras, 2000). Detectable levels of paroxetine were noted in all maternal serum samples and in 24 of the 25 breast milk samples. However, in all of the infant serum samples, the paroxetine concentrations were below the lower limit of quantification with the mean infant dose being $1.1 \%$ of the maternal dose, not unlike the data reported by Ohman et al. (1999). The authors rightfully pointed out that while there were no short-term adverse effects, future studies would be needed to address a more systematic method for observing and recording adverse effects, and future studies need follow-up assessments to evaluate potential long-term effects of paroxetine exposure.

In one of the only studies looking at infant development over a period of time (one year), and one of the few studies that included control groups, the paroxetine exposed group did not differ from the group who did not take paroxetine and did not breastfeed their infants or the group who breastfed their infants but did not take any drugs (Merlob, Stahl, \& Sulkes, 2004). The parents, pediatricians and nurses completed forms on infant weight and developmental milestones at ages three, six and twelve months. The paroxetine apparently did not affect weight gain or development in the infants. The authors, however, added a caveat that paroxetine would be an acceptable drug for depression in breastfeeding women assuming that they adhere to the lowest dose per day $(20 \mathrm{mg})$, that they took the dose at bed time, that they avoided combinations of drugs and that they ensured close medical follow-up of their infants.

\section{Fluoxetine (Prozac)}

Relatively higher concentrations have been noted for fluoxetine in breast milk and infant plasma. In an electronic search of MEDLINE and PsychInfo from 1966 to 2002, 57 studies of 
maternal plasma, breast milk and/or infant plasma antidepressant levels measured by liquid chromatography were identified (Weissman, Levy, Hartz, Bentler, Donohue, Ellingrod \& Wisner, 2004). The average infant-maternal plasma ratio was calculated for each drug, and correlations between infant plasma level and maternal dose, as well as between maternal plasma level and breast milk level were calculated. In the studies reviewed, paroxetine and sertraline effects were usually undetectable. Fluoxetine produced the highest proportion (22\%) of infant levels that were detected, and these were elevated to $10 \%$ of the average maternal level. Based on a smaller number of samples, citalopram-exposure produced elevated levels in $17 \%$ of infants. Thus, the authors concluded that paroxetine and sertraline may be the preferred choices by breastfeeding women, and they recommended limited use of fluoxetine and citalopram. A similar interpretation was made in a Norwegian study in which the authors suggested that fluoxetine should not be the first alternative, and high doses of citalopram should also be used with caution (Nordeng, Bergsholm, Bohler \& Spigset, 2001). However, their results were based on an extremely small sample $(\mathrm{N}=8)$.

In a study in which the maternal and infant transferable blockade was assessed, not unlike the study already described for paroxetine, (Epperson et al, 2001), platelet serotonin (5-HT) was measured in breastfeeding mother-infant pairs before and after four to twelve weeks of maternal fluoxetine treatment for postpartum depression (Epperson, Jatlow, Czarkowski \& Anderson, 2003). The infants were approximately 4-months at the start of the study. In this sample, all but one infant experienced little or no decline in whole-blood (platelet) 5-HT concentrations. These data suggested that most infants continued to breastfeed without experiencing clinically significant changes in platelet 5-HT transport while their mothers were being treated with 20 to $40 \mathrm{mg}$ of fluoxetine.

\section{Citalopram (Celexa)}

Citalopram has typically been the highest concentration SSRI antidepressant, when it has been compared to sertraline, paroxetine and fluoxetine. However, in studies that have only measured citalopram, negligible or no effects have been noted. For example, in a study in which one group who were depressed and were undergoing citalopram therapy were compared to a second group who were depressed but not undergoing citalopram therapy, and a third group that were healthy women (non-depressed and not taking medications), there were no statistically significant differences between groups on the rate of adverse events (Lee, Woo \& Ito, 2004). The problem, again, however, was the small sample size. In addition, the depressed women sample taking citalopram was three times the sample size of the group of depressed women who were not undergoing the therapy. Finally, as in most of these studies, there was no specification or no operational definition of adverse events.

In a second study that reported undetectable concentrations or at least low concentrations of citalopram $(\mathrm{N}=21)$, the citalopram and metabolite concentrations in the milk were 2 to 3 -fold higher compared with maternal plasma concentrations, but the infant citalopram and metabolite plasma concentrations were very low or undetectable (Heikkinen, Ekblad, Kero, Ekblad \& Laine, 2002). The authors also suggested that the infants' development was normal at one year of age.

\section{Methodological Problems}

The data presented in the form of empirical studies and reviews suggest that sertraline and paroxetine may be lower-concentration drugs than fluoxetine and citalopram when infant serum and breast milk are examined (Eberhard-Gran, Eskild \& Opjordsmoen, 2006). However, many of the comparison studies have potential confounding variables like severity and duration of depression and variability of drug timing and dosage. Further, as already mentioned, this 
literature is based primarily on case studies. Randomized controlled studies have not been performed, as is true for many antidepressant protocols, even in those studies that have treatment and no treatment groups. The failure to randomly assign and to stratify on confounding variables leads to unmatched groups. The dependent measures in these studies are also limited to breast milk and serum measures, thus making it difficult to determine the effects of the antidepressants on maternal depression itself and on infant growth and development. The studies on antidepressants effects on postpartum depression are found in a separate literature (Hoffbrand, Howard \& Crawley, 2001; Misri, Reebye, Corral \& Milis, 2004; Nonacs, Soares, Viguera, Pearson, Poitras \& Cohen, 2005). Although the drug exposure, in general, seems to be higher during pregnancy through placental passage than during the postpartum period through breast milk, no definitive conclusions can be made from a case study database. Certainly, controlled studies are needed. Some data also suggest that future studies can be conducted on breast milk alone given the strong correlation between breast milk concentrations and infant serum concentrations (Suri, Stowe, Hendrick, Hostetter, Widawski $\&$ Altshuler, 2002). Using breast milk alone would make these studies less invasive. Thus, these studies have limitations including, as already mentioned, the small sample sizes, the difficult problem of random assignment and the lack of control groups and long-term followup studies.

Other potentially confounding variables that have not been controlled, for example, severity and duration of depression, are factors that are not typically considered. In a study that highlighted that problem (Hendrick, Smith, Hwang, Altshuler \& Haynes, 2003), seventy-eight breastfeeding women taking antidepressant medications were followed and evaluated for maternal mood at 6,12, and 18 months postpartum, and their infants' weights were obtained from pediatric records. The infants' weights were not significantly different from the weights of 6-month-old breastfed infants from normative populations. However, for the mothers who relapsed for major depressive episodes lasting two months or more, the infant weights were significantly lower than the weights of infants whose mothers relapsed for brief depressive episodes of less than two months. Thus, although mere exposure to antidepressant medications did not appear to affect the infant weights, infants exposed to maternal depression lasting two months or more appeared to experience significantly lower weight gain than infants of those experiencing brief major depressive episodes. It is also possible that the women receiving the antidepressants were more severely depressed, and the more severe depression could confound these effects. The data from this study highlighted the many factors that interact with the assessment of antidepressant effects on breast milk, breastfeeding and infant development.

\section{Summary}

In summary, although a large literature supports the benefits of breastfeeding, this review suggests that breastfeeding is less common among postpartum depressed women, even though their infants benefit from the breastfeeding. Depressed mothers, in part, do not breastfeed because of their concern about potentially negative effects of antidepressants on their infants. Although sertraline and paroxetine concentrations are not detectable in infants, fluoxetine and citalopram do have detectable levels. Unfortunately these findings are not definitive because they are based on very small sample, uncontrolled studies. As in the literature on prenatal antidepressant effects, the question still remains whether the antidepressants or the untreated depression itself has more negative effects on the infant. Both antidepressants and untreated depression have notable negative effects.

It is also possible that the positive effects of breastfeeding may outweigh the positive effects of the antidepressants for both the mother and the infant. In addition, some alternative therapies may substitute or attenuate the effects of antidepressants such as vagal stimulation (Chambers \& Allen 2002) or massage therapy (Field et al, In press), both therapies being noted to reduce 
depression. Further studies are needed on the negative side effects of psychotropic medications during breastfeeding and on the side effects of abruptly removing this therapy (Gentile, $2005 b$ ). Studies of this kind and professional guidance on the course of therapy on a case-bycase basis are needed for the benefit of the breastfeeding mother and the breastfed infant.

\section{Acknowledgements}

This research was supported by a Merit Award (MH \# 46586), Senior Research Scientist Awards (MH\# 00331 and AT\# 001585) an NIH grant (\#AT00370) and a March of Dimes Grant (\# 12-FYO3-48) to Tiffany Field and funding from Johnson \& Johnson Pediatric Institute to the Touch Research Institutes. Correspondence and requests for reprints can be sent to Tiffany Field, Ph.D., Touch Research Institutes, University of Miami School of Medicine, PO Box 016820, Miami, Florida, 33101. Business phone number (305) 243-6781. E-mail tfield@med.miami.edu

\section{References}

Barone JG, Ramasamy R, Farkas A, Lerner E, Creenan E, Salmon D, Tranchell J, Schneider D. Breastfeeding During Infancy May Protect Against Bed-wetting During Childhood. Pediatrics 2006;118:254-9. [PubMed: 16818572]

Berle J, Steen V, Aamo T, Breilid H, Zahlsen K, Spigset O. Breastfeeding during maternal antidepressant treatment with serotonin reuptake inhibitors: infant exposure, clinical symptoms, and cytochrome $\mathrm{p} 450$ genotypes. Journal of Clinical Psychiatry 2004;65:1228-34. [PubMed: 15367050]

Chambers AS, Allen JJ. Vagal tone as an indicator of treatment response in major depression. Psychophysiology 2002;39:861. [PubMed: 12462513]

DiPrieto J, Larson S, Porges S. Behavioral and heart rate pattern differences between breast-fed and bottle-fed neonates. Developmental Psychology 1987;23:467-74.

Eberhard-Gran M, Eskild A, Opjordsmoen S. Use of psychotropic medications in treating mood disorders during lactation: practical recommendations. CNS Drugs 2006;20:187-98. [PubMed: 16529525]

Epperson N, Czarkowski K, Ward-O'Brien D, Weiss E, Gueorguieva R, Jatlow P, Anderson G. Maternal sertraline treatment and serotonin transport in breast-feeding mother-infant pairs. American Journal of Psychiatry 2001;158:16317.

Epperson N, Jatlow P, Czarkowski K, Anderson G. Maternal fluoxetine treatment in the postpartum period: effects on platelet serotonin and plasma drug levels in breastfeeding mother-infant pairs. Pediatrics 2003;112:425.

Field, T.; Diego, M.; Hernandez-Reif, M. Developmental Review. Massage Therapy Research. In Press

Field T, Hernandez-Reif M, Feijo L. Breastfeeding in depressed mother-infant dyads. Early Child Development and Care 2002;172:539-545.

Galler J, Harrison R, Biggs M, Ramsey F, Forde V. Maternal mood predicts breastfeeding in Barbados. Journal of Developmental and Behavioral Pediatrics 1999;20:80-87. [PubMed: 10219685]

Gentile S. SSRIs in pregnancy and lactation: emphasis on neirodevelopmental outcome. CNS Drugs 2005a;19:623-33. [PubMed: 15984897]

Gentile S. The safety of newer antidepressants in pregnancy and breastfeeding. Drug Safety 2005b; 28:137-152. [PubMed: 15691224]

Gerrish CJ, Mennella JA. Short-term influence of breastfeeding on the infants' interaction with the environment. Developmental Psychology 2000;36:40-48.

Grummer-Strawn LM, Mei Z. Does breastfeeding protect against pediatric overweight? Analysis of longitudinal data from the Centers for Disease Control and Prevention Pediatric Nutrition Surveillance System. Pediatrics 2004;113:e81-86. [PubMed: 14754976]

Hatton D, Harrison-Hohner J, Coste S, Dorato V, Curet L, McCarron D. Symptoms of postpartum depression and breastfeeding. Journal of Human Lactation 2005;21:444-9. [PubMed: 16280561]

Hart S, Boylan LM, Carroll S, Musick YA, Lampe RM. Brief report: Breast-fed one-week olds demonstrate superior neurobehavioural organisation. J Pediatric and Psychology 2003;28:529-34.

Heikkinen T, Ekblad U, Kero P, Ekblad S, Laine K. Citalopram in pregnancy and lactation. Clinical Pharmacological and Therapeutics 2002;72:184-91. 
Henderson J, Evan S, Straton J, Priest S, Hagan R. Impact of postnatal depression on breastfeeding duration. Birth 2003;30:175-80. [PubMed: 12911800]

Hendrick V, Fukuchi A, Altshuler L, Widawski M, Wertheimer A, Brunhuber M. Use of sertraline, paroxetine and fluvoxamine by nursing women. British Journal of Psychiatry 2001;179:163-6. [PubMed: 11483479]

Hendrick V, Smith L, Hwang S, Altshuler L, Haynes D. Weight gain in breastfed infants of mothers taking antidepressant medications. Journal of Clinical Psychiatry 2003;64:410-2. [PubMed: 12716242]

Hoffbrand S, Howard L, Crawley H. Antidepressant drug treatment for postnatal depression. Cochrane Database Syst Rev 2001;2:CD002018. [PubMed: 11406023]

Hoffman D, Birch E, Birch D, Uauy R, Castaneda Y, Lapus M, Wheaton D. Impact of early dietary intake ansd blood lipid composition of long-chain polyunsaturated fatty acids on later visual development. Journal of Pediatrics and Gastroenterology Nutrition 2000;31:540-53.

Hostetter A, Stowe Z, Cox M, Ritchie J. A novel system for the determination of antidepressant condetration in human breast milk. Therapeutic Drug Monitoring 2004;26:47-52. [PubMed: 14749550]

Howard LM, Hoffbrand S, Henshaw C, Boath L, Bradley E. Antidepressant prevention of postnatal depression. Cochrane Database Syst Rev 2005;2:CD004363. [PubMed: 15846711]

Jackson KM, Nazar A. Breastfeeding the immune response and long-term health. Health Journal of American Osteopath Association 2006;106:203-207.

Jones N, McFall B, Diego M. Patterns of brain electrical activity in infants of depressed mothers who breastfeed and bottle feed: the mediating role of infant temperament. Biological Psychology 2004;67:103-124. [PubMed: 15130527]

Kuzela A, Stifter C, Worobey J. Breastfeeding and mother-infant interactions. Journal of Reproductive and Infant Psychology 1990;8:185-94.

Labbok MH, Wardlaw T, Blanc A, Clark D, Terreri N. Trends in exclusive breastfeeding: findings from the 1990s. Journal of Human Lactation 2006;22:272-6. [PubMed: 16885487]

Lavelli M, Poli M. Early mother-infant interaction during breast- and bottle-feeding. Infant Behavior and Development 1998;21:667-84.

Lee A, Woo J, Ito S. Frequency of infant adverse events that are associated with citalopram use during breast-feeding. American Journal of Obstetrics and Gynecology 2004;190:218-21. [PubMed: 14749663]

Merlob P, Stahl B, Sulkes J. Paroxetine during breast-feeding: infant weight gain and maternal adherence to counsel. European Journal of Pediatrics 2004;163:135-9. [PubMed: 14745552]

Misri S, Kim J, Riggs K, Kostaras X. Paroxetine levels in postpartum depressed women, breast milk, and infant serum. Journal of Clinical Psychiatry 2000;61:828-32. [PubMed: 11105735]

Misri S, Kostaras X. Benefits and risks to mother and infant of drug treatment for postnatal depression. Drug Safety 2002;25:903-11. [PubMed: 12381212]

Misri S, Reebye P, Corral M, Milis L. The use of paroxetine and cognitive-behavioural therapy in postpartum depression: A randomized controlled trial. Journal of Clinical Psychiatry 2004;65:123641. [PubMed: 15367052]

Nonacs RM, Soares CN, Viguera AC, Pearson K, Poitras JR, Cohen LS. Bupropion SR for the treatment of postpartum depression: a pilot study. International Journal of Neuropsychopharmacology 2005;8:445-9. [PubMed: 15817137]

Nordeng H, Bergsholm Y, Bohler E, Spigset O. The transfer of selective serotonin reuptake inhibitors to human milk. Tidsskrift for den Norske Laegeforening 2001;121:199-203. [PubMed: 11475200]

Oddy WH, Scott JA, Graham KI, Binns CW. Breastfeeding influences on growth and health at one year of age. Breastfeed Review 2006;14:15-23.

Ohman R, Hagg S, Carleborg L, Spigset O. Excretion of paroxetine into breast milk. Journal of Clinical Psychiatry 1999;60:519-23. [PubMed: 10485633]

Sacker A, Quigley M, Kelly Y. Breastfeeding and developmental delay: Findings from the Millennium Cohort Study. Pediatrics 2006;118:e682-e689. [PubMed: 16950960] 
Stowe Z, Cohen L, Hostetter A, Ritchie J, Owens M, Nemeroff C. Paroxetine in human breast milk and nursing infants. American Journal of Psychiatry 2000;157:185-9. [PubMed: 10671385]

Stowe Z, Hostetter A, Owen M, Ritchie J, Sternberg K, Cohen L, Nemeroff C. The pharmacokinetics of sertraline excretion into human breast milk: determinants of infant serum concentrations. Journal of Clinical Psychiatry 2003;64:73-80. [PubMed: 12590627]

Suri R, Stowe Z, Hendrick V, Hostetter A, Widawski M, Altshuler L. Estimates of nursing infant daily dose of fluoxetine through breast milk. Biological Psychiatry 2002;52:446-51. [PubMed: 12242061]

Shields L, O'Callaghan M, Williams GM, Najman JM, Bor W. Breastfeeding and obesity at 14 years: a cohort. study. J Paediatr Child Health 2006;42:289-296. [PubMed: 16712560]

Taj R, Sikander K. Effects of maternal depression on breast-feeding. Journal of the Pakistan Medical Association 2003;53:8-11. [PubMed: 12666844]

Uvnas-Moberg K, Petersson M. Oxytocin, a mediator of anti-stress, well-being, social interaction, growth and healing. Z Psychosomatic Medicine and Psychotherapy 2005;51:57-80.

VanDiver T. Relationship of mothers' perceptions and behavior to the duration of breastfeeding. Psychological Reports 1997;80:1375-1384. [PubMed: 9246903]

Weissman A, Levy B, Hartz A, Bentler S, Donohue M, Ellingrod V, Wisner K. Pooled analysis of antidepressant levels in lactating mothers, breast milk, and nursing infants. American Journal of Psychiatry 2004;161:1066-78. [PubMed: 15169695]

Whitby D, Smith K. The use of tricyclic antidepressants and selective serotonin reuptake inhibitors in women who are breastfeeding. Pharmacotherapy 2005;25:411-25. [PubMed: 15843288]

Worobey J. Feeding method and motor activity in 3-month-old human infants. Perceptual and Motor Skills 1998;86:883-895. [PubMed: 9656283]

Zeskind P, Marshall T, Goff D. Rhythmic organization of heart rate in breast-fed and bottle-fed newborn infants. Early Development and Parenting 1992;1:79-87.

Infant Behav Dev. Author manuscript; available in PMC 2009 September 1. 